\title{
IRSETCONF
}

\section{PREPARATION AND CHARACTERIZATION OF HYDROXYPROPYLCELLULOSE/GRAPHENE OXIDE NANOCOMPOSITE MACROPOROUS CRYOGELS}

\author{
Georgi L. Georgiev, Maya Staneva and Petar D. Petrov \\ Institute of Polymers, Bulgarian academy of sciences, "Akad. G. Bonchev" St. 103A, 1113 Sofia, Bulgaria
}

\begin{abstract}
ABSTARCT
In the past few years, graphene and graphene based materials have attracted great interest due to their unique electronic, mechanical, optical, and thermal properties. Graphene oxide (GO) has all the advantages of graphene, but, unlike it, can be dispersed well in water. Polymeric cryogels are fascinating soft materials with wide applications in drug delivery, tissue engineering, chromatography, etc. Nanocomposite cryogel materials from natural polysaccharides and GO are attractive because they possess a set of favourable properties like biodegradability and biocompatibility, high porosity and water uptake, improved mechanical strength and electrical conductivity, and could broad the range of applications in the area of medicine and biotechnology.

In this contribution we report on the fabrication of novel supermacroporous nanocomposite cryogels based on hydroxpropylcellulose (HPC) and graphene oxide by two different methods. The first method involves mixing of aqueous dispersion of GO and solution of HPC, freezing and photo-crosslinking. In this case, GO is embedded into the cryogel walls. In the second method, a pre-formed and lyophilized HPC cryogel is immersed in dispersion of GO and then frozen and lyophilized. Thus GO is deposited on the inner surface of cryogel. The second method allows the preparation of super-macroporous nanocomposites of a higher GO content, better control of physico-mechanical properties and enhanced electrical conductivity ( 3 orders of magnitude higher than pure HPC) as compared to the nanocomposite cryogels made by the first method.
\end{abstract}

Keyword: biopolymer, cryogel, graphene oxide 